\title{
Du verbe au nom : calques et décalages aspectuels
}

\author{
Haas, Pauline*, Huyghe, Richard**, \& Marín, Rafael* \\ *Université Lille 3 - CNRS UMR 8163 («STL ») \\ **Université Paris 7 \\ paulineha@numericable.fr, rhuyghe@free.fr, rafael.marin@univ-lille3.fr
}

\section{Introduction}

Ce travail s'inscrit dans un projet de recherche visant à déterminer les propriétés sémantiques, en particulier aspectuelles, des nominalisations en français. Nous prenons ici une définition minimale de nominalisation, à caractère morphologique : par nominalisation, nous désignons tout nom dérivant d'un verbe.

L'aspect est envisagé ici dans sa composante lexicale, et non grammaticale. Il va de soi que ces deux composantes interagissent en discours selon des règles étudiées par ailleurs (voir par exemple Verkuyl 1971, Dowty 1979, Smith 1991). Il n'en reste pas moins que le calcul de l'aspect en contexte se fait à partir d'un prédonné lexical inscrit dans le sens des unités en jeu. Nous postulons que ce prédonné peut, en tant que tel, faire l'objet d'une étude à part entière.

Notre enquête prend pour point de départ les travaux consacrés aux propriétés aspectuelles des verbes, dont beaucoup sont fondés sur la classification vendlerienne entre états, activités, accomplissements et achèvements. Cette classification nous fournit une première piste de réflexion : les catégories de Vendler sont-elles transposables dans le domaine nominal ? Plus précisément, la spécification des traits primitifs de dynamicité, de délimitation et de durée, qui permettent de distinguer entre états, activités, accomplissements et achèvements, se retrouve-t-elle dans le domaine nominal ? Et si oui, verbes et noms correspondants ont-ils les mêmes caractéristiques aspectuelles ?

Il s'agit de déterminer si dans le processus de nominalisation des verbes il y a un transfert des propriétés aspectuelles. Cela demande de savoir comment se manifeste l'aspect dans le domaine nominal, et de disposer de tests adéquats pour en cerner les spécifications. Plus généralement, l'étude des nominalisations de verbes permet de s'interroger sur les critères de signification communs aux catégories verbale et nominale, et sur la «porosité » sémantique de ces deux catégories.

Nous nous intéresserons tout d'abord à la transposition du trait statique / dynamique, qui dans le domaine verbal permet de distinguer entre états et actions. Puis nous tenterons d'établir une première série de distinctions dans le domaine actionnel, en tenant compte des propriétés de délimitation et de durée des situations dénotées. Apparaîtra en filigrane un principe général de catégorisation des noms concernés, fondé sur le rapport au temps et ses modalités. Il sera établi (i) que les traits aspectuels élémentaires s'appliquent aux nominalisations et (ii) que l'aspect des nominalisations, quoique souvent fidèle à celui des verbes dont elles dérivent, n'est pas toujours calqué sur lui. A cet égard, nous accorderons une attention particulière aux nominalisations de verbes dont l'aspect lexical est multiple ou sous-déterminé.

Précisons d'emblée que les déverbaux à valeur concrète (agentive, résultative, instrumentale, etc.) ${ }^{1}$ sont exclus de notre champ d'étude, étant donné qu'ils n'ont pas de propriétés aspectuelles - a fortiori, la question de leur héritage aspectuel ne se pose pas (cf. Huyghe \& Marín 2007, Haas \& Huyghe à paraître). Les cas pertinents sont les nominalisations susceptibles de dénoter des situations, qu'il s'agisse d'actions, d'états ou d'événements. 


\section{Noms statiques vs noms dynamiques}

Le critère aspectuel de la staticité / dynamicité, qui structure en amont les différentes catégories aspectuelles, est valide dans le domaine nominal. La différence entre noms d'états et noms d'actions apparaît notamment dans l'expression de la caractérisation temporelle. La dynamicité implique en effet le rapport au temps, les situations dynamiques étant dotées d'une extension et/ou d'un ancrage temporel. Selon ce principe, un nom d'action doit pouvoir apparaître dans au moins une des trois tournures suivantes :
a. un $\mathrm{N}$ de x temps (e.g. un $N$ de deux minutes, un $N$ de deux heures, un $N$ de deux jours)
b. x temps de $\mathrm{N}$ (e.g. deux minutes de $N$, deux heures de $N$, deux jours de $N$ )
c. le t du N (e.g. la date $d u N$, le moment $d u N$, l'instant $d u N$ )

Corollairement, un nom qui ne peut figurer dans aucune de ces tournures est statique ${ }^{2}$. Tel est le cas des noms suivants :
a. *une (croyance / préférence / signification / possession / ressemblance / volonté) de deux (minutes / heures / jours) b. *deux (minutes / heures / jours) de (croyance / préférence / signification / possession / ressemblance / volonté)
c. *(la date / le moment / l'instant) de la (croyance / préférence / signification / possession / ressemblance / volonté)

Croyance, préférence, signification, etc. sont des noms abstraits atemporels, au sens où leur dénotation n'a pas de relation directe avec le temps. Ces noms décrivent des propriétés (signification, ressemblance), des qualités (volonté), des aptitudes intellectuelles (croyance, préférence), etc. Ils sont dérivés de verbes d'état et leur sont donc fidèles du point de vue aspectuel. Par leur staticité, ils se distinguent des noms abstraits dérivés de verbes d'action, qui eux sont dynamiques.

De fait, croyance, préférence, signification, etc. contrastent avec des noms comme réunion, bricolage, accouchement, lecture, présentation ou atterrissage, compatibles avec les expressions sous (1) :
a. une présentation de deux minutes, une réunion de deux heures
b. deux heures de bricolage, deux jours de lecture
c. la date de l'accouchement, le moment de l'atterrissage

Cette compatibilité peut s'expliquer par la dynamicité des noms testés, elle-même héritée de celle des verbes correspondants. Que réunion, bricolage, accouchement, etc. soient dynamiques est confirmé par le fait que ces noms valident au moins une des conditions suivantes (cf. Haas \& Huyghe à paraître) :

- $\quad$ le nom peut s'employer dans l'expression un $N$ en cours ou en cours de $N$ (cf. Anscombre 2005, 2007) : en cours d'accouchement, une réunion en cours ;

- le nom se construit avec un verbe support, dans un emploi synonyme ou quasi-synonyme du verbe dont il est dérivé (cf. Giry-Schneider 1978, Gross 1996) : faire du bricolage, procéder à un atterrissage (proches de bricoler et atterrir);

- le nom admet une structure argumentale complexe incluant un agent introduit par par (cf. entre autres Grimshaw 1990, Alexiadou 2001) : la lecture du verdict par le président, la présentation du rapport par Sophie.

Chacune de ces conditions est suffisante pour montrer qu'un nom est dynamique. On observe que les noms d'états ne vérifient aucun des tests cités, ce qui est conforme à leur non dynamicité. 


\section{Activités et événements}

Nous n'avons pas pour l'instant distingué entre les noms d'actions selon qu'ils acceptent les constructions d'extension et/ou d'ancrage temporels. Différentes classes de nominalisations dynamiques peuvent être dégagées à partir de la variation de ce double critère.

\subsection{La spécificité des noms d'activités}

Il faut faire une première distinction entre les noms dynamiques qui dénotent des situations datables (i.e. dotées d'un ancrage temporel) et ceux qui dénotent des situations non datables :

(4) a. la date (du cambriolage / de l'intervention), le moment (de la préparation / de la réunion), l'instant (de l'explosion / du démarrage)

b. ??la date (de la natation / du jonglage), ??le moment (du jardinage / du braconnage), ??1'instant (du patinage / du canotage)

Les noms sous (4b) n'admettent que l'extension temporelle, dans la tournure $x$ temps de $N$ :

(5) deux minutes de (patinage / jonglage), deux heures de (natation / canotage), deux jours de (jardinage / braconnage)

Ici, l'alternance $x$ temps de $N$ / un $N$ de $x$ temps n'est pas possible, alors qu'elle peut l'être pour d'autres noms d'actions (e.g. une discussion de dix minutes / dix minutes de discussion) :

??(un patinage / un jonglage) de deux minutes, ??(une natation / un canotage) de deux heures, ??(un jardinage / un braconnage) de deux jours

La raison en est que natation, jonglage, etc. sont des noms massifs (cf. Flaux \& Van de Velde 2000, Heyd \& Knittel 2006, Haas \& Huyghe à paraître). En effet, on dit (7a) et non (7b) :

a. de la natation, du patinage, du braconnage, du jardinage, du jonglage, du canotage

b. ??des natations, ??deux patinages, ??trois braconnages, ??quelques jardinages, ??plusieurs jonglages, ??différents canotages

En tant que noms massifs, natation, jonglage, etc. décrivent des entités homogènes et non délimitées, dont on peut prélever une certaine quantité, mais qui n'en spécifient pas une — d'où le contraste observé dans (5) vs (6). Ils s'emploient particulièrement bien avec le verbe support faire : faire (de la natation / du patinage / du jardinage / etc.).

L'absence de délimitation décrite par ces noms est confirmée par le fait qu'ils ne se combinent pas avec pendant. L'expression pendant le $N$ implique en effet la description d'un intervalle fermé (cf. Berthonneau 1989) :
a. ??Sylvain a cassé plusieurs massues pendant le jonglage
b. ??J'ai eu une crampe pendant le canotage
c. ??Pierre s'est perdu pendant le braconnage

De même, natation, jonglage et les autres ne s'emploient pas avec en cours de, qui présuppose la délimitation temporelle (cf. Anscombre 2007) :

(9) a. ??Je me suis blessé en cours de jardinage

b. ??Marion est tombée en cours de patinage 
Les phrases (8) ne sont éventuellement acceptables que si l'on comprend le $N$ comme « une séance de $\mathrm{N}$ », c'est-à-dire si l'on contraint contextuellement la lecture comptable du nom (e.g. Pendant la natation, plusieurs triathlètes ont eu des crampes, Il s'est endormi pendant le patinage). De même, les phrases sous (9) sont correctes si en cours de n'est pas pris comme locution prépositionnelle, mais dans le sens de « pendant la leçon de ».

Ainsi, natation, jonglage, etc. décrivent des actions atéliques, c'est-à-dire sans délimitation intrinsèque. Ils correspondent à ce que, dans le domaine verbal, on catégorise comme des «activités » ou des « processus » (cf. Vendler 1967, Mourelatos 1978, Smith 1999). Précisément, ces noms sont tous dérivés de verbes d'activité ; ils en héritent donc l'aspect dynamique non délimité. Nous laissons pour l'instant en suspens la question de savoir si cet héritage est généralisé et si tous les noms abstraits dérivés de verbes d'activité dénotent eux-mêmes des activités.

L'absence de délimitation temporelle décrite par natation, jonglage, etc. explique pourquoi ces noms ne peuvent pas se voir associer de datation (4b). Ils décrivent des types d'actions, plutôt que des occurrences (i.e. des actions spécifiques, spatio-temporellement individuées). De fait, natation, jonglage, etc. ne peuvent pas référer à des événements. Ils s'emploient difficilement avec avoir lieu et le lieu du $N$, qui sélectionnent des noms d'événements (cf. Huyghe 2006) :
a. ??(La natation / le braconnage / le canotage) a eu lieu ce matin
b. ??le lieu (du jardinage / du jonglage / du patinage)

(10b) est à mettre en parallèle avec (4b) : non occurrentiels, les noms d'activités ne dénotent pas des situations particulières, inscrites dans l'espace-temps. Leur interprétation avec l'article défini est essentiellement générique (cf. Heyd \& Knittel 2006, Haas \& Huyghe à paraître), comme dans les exemples suivants :

(11) a. (Le jardinage / la natation / le jonglage), c'est agréable

b. Sylvain est un adepte (du patinage / du canotage / du braconnage)

(12) a. La loi sanctionne durement le braconnage

b. Il y a beaucoup de sites Internet consacrés au jardinage

c. Ce spectacle mêle la danse et le jonglage

d. Le canotage a depuis longtemps cédé la place au kayak et à l'aviron

e. Les enfants regardent une émission sur le patinage

f. La natation est un sport qui comporte très peu de contre-indications

Tout élément qui viendrait spécifier la situation, dans une visée occurrentielle ou événementielle, pose problème. Aussi, quand les noms du type natation se construisent avec un complément adnominal en de renvoyant à un de leurs arguments, celui-ci doit-il exprimer un actant générique ou habituel. Le nom tête reçoit alors une interprétation variétale, indiquant un sous-type de l'activité dénotée (e.g. On essaie d'encourager la natation des tout petits, Le braconnage des éléphants porte essentiellement sur l'ivoire et la viande).

Il ressort de l'ensemble de nos observations que les activités, à travers leurs nominalisations, se présentent comme des types d'actions qui par essence durent, mais qui n'ont ni délimitation ni ancrage spécifique dans l'espace-temps. 


\subsection{L'hétérogénéité des noms d'événements}

Aux noms d'activités, massifs, s'opposent les noms d'actions comptables :

(13) des accouchements, plusieurs discussions, quelques promenades, trois assassinats, dix interventions, différents tournages

En tant que noms dynamiques comptables, accouchement, discussion, etc. impliquent une délimitation temporelle. Ils sont datables (e.g. la date de (l'accouchement / l'assassinat / l'intervention), le moment (de la discussion / de la promenade / du tournage)) et peuvent se construire avec pendant :

(14) a. Ils ont beaucoup parlé pendant la promenade

b. Pierre s'est évanoui pendant l'accouchement

c. Plusieurs pompiers se sont blessés pendant l'intervention

La signification d'une délimitation leur permet de dénoter des occurrences d'actions, et notamment des événements. Ainsi peuvent-ils être sujet de avoir lieu :
a. Le tournage aura lieu à l'automne 2008
b. La discussion a lieu dans le bureau du directeur
c. L'assassinat a eu lieu à proximité du domicile de la victime

Et de même qu'ils peuvent figurer dans la date $d u N$, ils sont compatibles avec le lieu $d u N$ :

(16) le lieu (de l'accouchement / du tournage / de la promenade / de la discussion / de l'assassinat / de l'intervention)

Les noms d'actions comptables se distinguent donc des noms d'activités par leur capacité à décrire des situations particulières. Les propriétés communes mises en évidence dans (13)-(16) ne doivent cependant pas cacher leur disparité.

\subsubsection{Occurrences ponctuelles}

Certains noms d'événements sont plus rétifs que d'autres à la complémentation d'étendue. En dépit de leur dynamicité, il est difficile de leur associer une expression de durée. D'une part, ils se construisent difficilement avec une expansion temporelle :

(17) ??un assassinat de deux heures, ??une condamnation de trois minutes, ??une découverte de six mois, ??un empoisonnement de quinze minutes, ??une naissance de cinq heures, ??une noyade de trois minutes

D'autre part, ils ne peuvent pas être sujets de durer :
a. ??L'assassinat a duré deux heures
b. ??La naissance a duré cinq heures
c. ??La découverte a duré six mois

Ces noms, alors qu'ils se combinent avec avoir lieu, ne sont pas non plus compatibles avec se dérouler, qui met en profil la durée des procès :

(19) a. ??La condamnation s'est déroulée au tribunal de commerce
b. ??La noyade s'est déroulée dans une zone de baignade non surveillée 


\section{c. ??L'empoisonnement s'est déroulé dans un grand hôtel londonien}

Il est difficile de discerner et de saisir différentes phases dans les actions décrites ici. Assassinat, naissance, etc. ne s'emploient pas comme sujets de prédicats inchoatifs ou terminatifs :
a. ??(L'assassinat / la découverte / la noyade) vient de commencer
b. ??(La naissance / la condamnation / l'empoisonnement) est bientôt terminé(e)

Ils se combinent également mal avec en cours de, qui appréhende le procès dans son déroulement (cf. Anscombre 2007) :
a. ??Le chirurgien est arrivé en cours de naissance
b. ??L'espion est en cours d'empoisonnement
c. ??Les secours sont intervenus en cours de noyade

Assassinat, naissance et les autres se présentent donc comme des noms d'actions ponctuelles. Ils correspondent aspectuellement à la catégorie des achèvements. Dérivés de verbes traditionnellement considérés comme relevant de cette catégorie, ils conservent la spécification aspectuelle de leurs bases.

L'existence des achèvements est souvent contestée dans le domaine verbal (cf. Mourelatos 1978, Verkuyl 1989), notamment parce que les actions considérées comme ponctuelles peuvent être appréhendées en extension (cf. Pierre est en train (d'atteindre le sommet / d'annuler son voyage / de passer la frontière / etc.)). Le même genre d'observation vaut dans le domaine nominal, puisque des noms comme annulation, explosion, rupture, arrestation, disparition, dérivés de verbes considérés comme des achèvements, valident certains des tests ci-dessus (e.g. L'arrestation s'est déroulée sans incident, Cette procédure est en cours d'annulation).

Certains faits linguistiques tendent néanmoins à montrer que des verbes comme annuler, découvrir, démarrer, etc. ne se comportent pas comme des accomplissements standards (cf. Rothstein 2004). Le fait que certains noms d'événements soient particulièrement réfractaires aux caractérisations d'étendue, ainsi que nous l'avons vu dans (17)-(21), permet de penser qu'il en va de même dans le domaine nominal. On peut y voir une confirmation de l'existence d'un pôle aspectuel correspondant aux occurrences ponctuelles. En effet, il est nécessaire de disposer de la catégorie des achèvements pour pouvoir rendre compte des faits recensés ci-dessus et, plus généralement, de la diversité de structure des événements.

\subsubsection{Occurrences culminantes et non culminantes}

Contrastant avec les noms d'événements ponctuels, certaines nominalisations décrivent sans contrainte des occurrences duratives. Ces noms peuvent figurer en position de $\mathrm{N}$ dans un $N$ de $x$ temps, le $N$ a duréx temps :

(22) un accouchement de six heures, une discussion de quarante minutes, une réparation de quinze minutes, une traversée de deux heures, une rénovation de plusieurs mois, une manifestation de trois heures

(23) a. La réparation a duré quinze minutes

b. La traversée a duré deux heures

c. La rénovation a duré plusieurs mois

Les procès décrits peuvent sans difficulté être saisis dans leur déroulement. (24)-(26) s'opposent ainsi à (19)-(21) : 
(24) a. La manifestation s'est déroulée en province

b. L'accouchement s'est déroulé dans un hôpital bruxellois

c. La discussion s'est déroulée dans le hall de l'hôtel

a. (L'accouchement / la manifestation / la rénovation) vient de commencer

b. (La discussion / la traversée / la réparation) est bientôt terminée

a. Pierre est arrivé en cours de discussion

b. Ce bâtiment est en cours de rénovation

c. La césarienne peut être programmée à l'avance ou décidée en cours d'accouchement

Les noms d'événements duratifs ont différentes origines aspectuelles. Certains, comme accouchement, sont dérivés de verbes d'accomplissement, c'est-à-dire de verbes décrivant des actions duratives et intrinsèquement délimitées; ils héritent donc directement des propriétés aspectuelles de leurs bases. D'autres, comme réparation, proviennent de verbes dont l'aspect change selon la délimitation de l'objet (cf. réparer une voiture vs réparer des voitures) - nous reviendrons dans la suite sur la question de leur fidélité aspectuelle. D'autres encore, et c'est plus surprenant, sont issus de verbes d'activité. Tel est le cas de discussion, manifestation, mais aussi de promenade, bombardement, simulation, etc. En effet, les verbes d'origine de ces noms sélectionnent régulièrement des compléments de temps en pendant et non en en, y compris dans le cas où ils ont un objet délimité :

a. Ils ont manifesté (pendant quatre heures / *en quatre heures)

b. Ils ont bombardé (une ville / des villes) (pendant deux jours / *en deux jours)

Dérivés de verbes atéliques, discussion, promenade, manifestation, bombardement, simulation, etc., parce qu'ils peuvent dénoter des actions délimitées dans le temps, sont en décalage avec leurs verbes d'origine. Ils se distinguent d'ailleurs nettement des purs noms d'activités, massifs et non occurrentiels, comme jonglage et natation, qui eux sont fidèles à leurs bases verbales (cf. § 3.1.).

Il y a pourtant une différence entre discussion, manifestation, etc. et les accomplissements prototypiques. Un nom comme accouchement, en effet, décrit un procès structuré, doté d'un point culminant marquant la réalisation de l'action. C'est cette structure qui à la fois implique le terme de l'action et le fixe. Dans le cas de discussion, manifestation, etc., les procès décrits ont certes une borne finale, mais l'existence et la spécification de cette borne ne sont pas imposées par la nature de l'action : il n'y a pas de point culminant consubstantiel au procès. En d'autres termes, la borne finale n'est pas fixée « de l'intérieur », selon une structure actionnelle : le procès se déroule de façon homogène et s'arrête à un moment (non spécifié) (cf. Haas \& Huyghe à paraître).

La différence entre ces deux types d'événements duratifs apparaît si l'on applique aux nominalisations concernées le test du «paradoxe imperfectif» (cf. Dowty 1979). Alors que l'interruption d'un accouchement bloque la réalisation de l'action, conformément à ce qu'on observe pour les accomplissements verbaux, l'interruption d'une discussion, d'une manifestation ou d'une promenade n'empêche pas l'action de s'être réalisée, comme c'est le cas pour les activités :

(28) L'accouchement a pu être interrompu au bout d'une heure > Elle n'a pas accouché

(29) a. La manifestation a été interrompue au bout de deux heures $>$ Ils ont manifesté

b. La discussion a été interrompue au bout d'une demi-heure > Ils ont discuté

c. La promenade a été interrompue par la pluie > Ils se sont promenés

Ainsi, discussion, promenade, etc., tout en appartenant à une catégorie aspectuelle différente de celle de leurs verbes d'origine, conservent une trace de l'aspect des verbes en question. Ils ont en effet en commun avec leurs bases de décrire des procès homogènes : ils indiquent une délimitation temporelle, 
mais pas de climax déterminant le terme de l'action. Les noms d'événements duratifs doivent donc être appréhendés comme une classe aspectuelle hétérogène.

\section{Noms dérivés de verbes sous-déterminés ou polysémiques}

Se dégage des observations précédentes un schéma de classification rudimentaire. La dénotation des nominalisations de sens abstrait se divise comme suit :
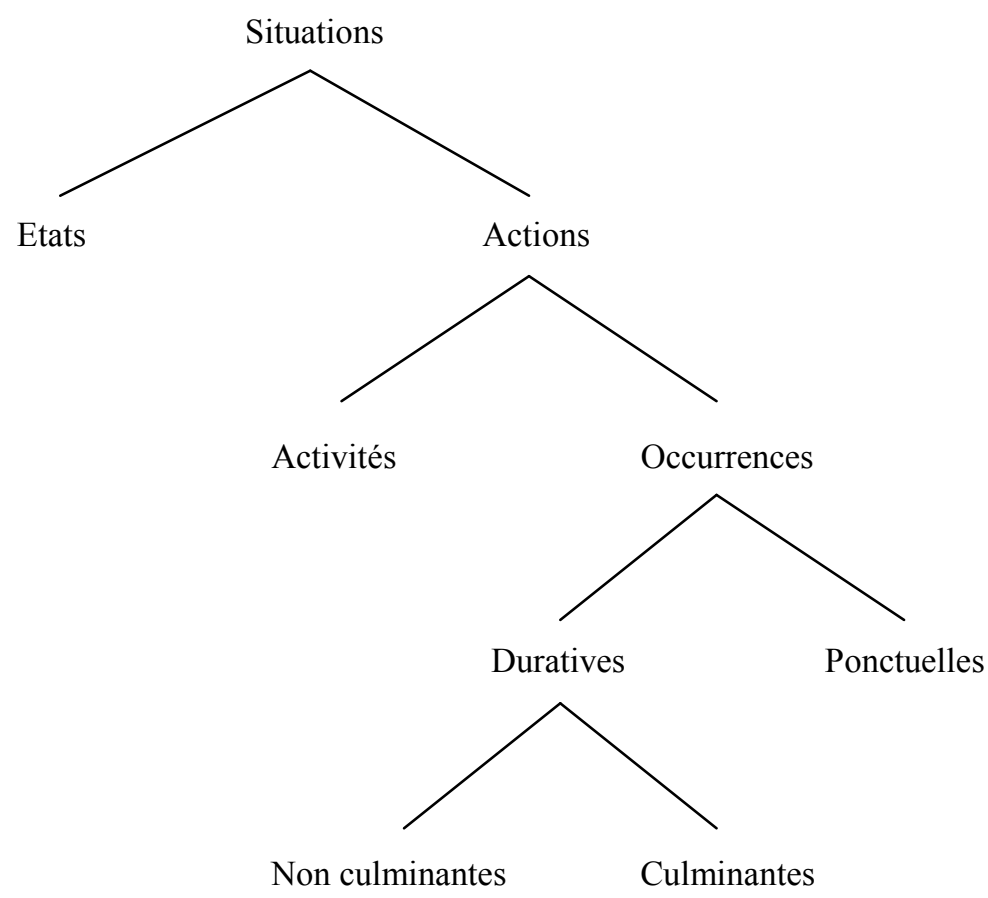

Ce schéma est assez proche de ceux traditionnellement établis dans le domaine verbal (voir par exemple Mourelatos 1978, Bach 1986, Verkuyl 1989). Du point de vue aspectuel, les nominalisations sont souvent fidèles à leurs verbes de base : elles en héritent les traits principaux. Nous avons cependant constaté l'existence de décalages sémantiques, puisque certains items lexicaux, selon qu'ils sont sous forme verbale ou nominale, relèvent de catégories aspectuelles différentes. Tel est le cas notamment des noms d'occurrences non culminantes, qui sont dérivés de verbes d'activité.

Pour approfondir l'examen de la fidélité aspectuelle entre les verbes et leurs dérivés nominaux, il faut s'interroger sur les nominalisations des verbes dont l'aspect lexical est considéré comme sous-déterminé, ou multiple (en cas de polysémie marquée). Nous nous pencherons ici sur quelques cas emblématiques.

\subsection{Nominalisations de verbes sous-déterminés}

Il est bien connu que de nombreux verbes transitifs sont sous-déterminés du point de vue aspectuel. Leur aspect [ \pm délimité] dépend de leur argument interne, et se calcule donc au niveau du SV (cf. entre autres Verkuyl 1989, Ghiglione 1990). Cette règle vaut en particulier dans le cas des accomplissements. Ainsi, des verbes comme réparer, cambrioler, privatiser, qui avec un objet délimité décrivent des accomplissements, se dotent d'une lecture d'activité dès lors que l'argument interne n'est plus clairement 
circonscrit - notamment lorsque les SN sont déterminés par l'article indéfini pluriel ou partitif. En effet, le verbe se construit sans difficulté avec un complément de temps en en dans le premier cas, mais pas dans le second:
a. Le garagiste a réparé la voiture en trois quarts d'heure
b. *Le garagiste a réparé des voitures en deux heures

Alors que réparer la voiture décrit un accomplissement standard, réparer des voitures est un prédicat d'activité : la non délimitation de l'objet (assimilable à un SN massif) se transmet à l'ensemble du SV. Cambrioler et privatiser se comportent à cet égard comme réparer : cambrioler (l'appartement / *des appartements) en deux heures, privatiser (l'entreprise /*des entreprises) en six semaines.

Qu'advient-il des nominalisations de ces verbes sous-déterminés? Dans le cas où l'objet est explicitement mentionné, i.e. lorsqu'il figure dans une structure argumentale, la nominalisation se conforme au verbe : l'aspect du SN se calque sur celui du SV correspondant, et on retrouve la double possibilité d'interprétation. Ainsi, avec un argument interne délimité, la nominalisation dénote un procès délimité, i.e. une occurrence, et non une activité. Elle est compatible avec la date $d u N$, pendant le $N$ et le Na eu lieu :
a. Quelle est la date de la privatisation de l'entreprise ?
b. Le garagiste s'est blessé pendant la réparation de la voiture
c. Le cambriolage de l'appartement a eu lieu le 5 novembre $^{3}$

Lorsqu'en revanche l'argument interne n'est pas délimité, le SN peine à dénoter une occurrence :
a. ??Quelle est la date de la privatisation d'entreprises ?
b. ??Le garagiste s'est blessé pendant la réparation de voitures
c. ??Le cambriolage d'appartements a eu lieu le 5 novembre

La non délimitation de l'objet se répercute sur la nominalisation, qui ne peut dénoter une entité temporellement délimitée, ni donc un événement. Le SN formé n'est pas spécifique ; il reçoit plutôt une interprétation générique, caractéristique des activités :

(33) a. La réparation de voitures anciennes nécessite un savoir-faire que peu de garagistes ont

b. La privatisation d'établissements publics ne peut se faire sans négociation préalable

c. Cette bande s'est spécialisée dans le cambriolage d'appartements bourgeois

Dans ce cas, la nominalisation prend valeur de nom d'activité. Comme pour les noms d'activités, l'alternance $x$ temps de $N$ / un $N$ de $x$ temps est impossible (cf. (5)-(6)) :

(34) a. Dix ans de privatisation d'établissements publics ont considérablement changé la structure du marché

b. ??Une privatisation d'établissements publics de dix ans a considérablement changé la structure du marché

(35) a. Après cinq ans de cambriolage d'appartements bourgeois, ils sont partis vivre aux Marquises

b. ??Après un cambriolage d'appartements bourgeois de cinq ans, ils sont partis vivre aux Marquises

Même l'emploi avec faire du est parfois possible (e.g. Dans ce garage, on fait de la réparation de voitures anciennes). Notons que dans (33)-(35), la nécessité de caractériser plus précisément l'objet en lui adjoignant un épithète est symptomatique de la lecture d'activité : on cherche à dénoter un certain type d'action correspondant à une sous-classe de l'activité décrite par le nom tête (cf. § 3.1.). 
Ainsi, les nominalisations de verbes sous-déterminés connaissent la même variation aspectuelle que leurs bases, suivant la délimitation de leur argument interne. L'aspect [ \pm délimité] est déterminé en contexte et il est conforme à celui de la forme verbale nominalisée.

On peut se demander si la double interprétation d'occurrence / activité perdure lorsque le nom s'emploie sans structure argumentale, dans des SN de la forme le $N$ sans expansion. Dans ce type d'emploi, la lecture occurrentielle est régulièrement permise :

a. La réparation a pris plus de trois heures

b. Depuis la privatisation, le prix de l'action a été multiplié par cinq

c. Après le cambriolage, nous avons fait installer une alarme

Le $N$ sans exp. est ici spécifique et anaphorique. Il y a un argument interne sous-entendu, qui est délimité. Autrement dit, le déverbal nominalise un accomplissement (e.g. réparer la voiture, privatiser l'entreprise, cambrioler la maison), sur lequel il se calque aspectuellement. Beaucoup de noms d'événements duratifs sont de ce type et nominalisent un accomplissement en intégrant un objet délimité implicite. Tel est le cas des dérivés de verbes sous-déterminés rencontrés au $§ 3.2 .2$. (réparation, mais aussi rénovation et traversée).

La lecture générique d'activité est elle aussi possible (e.g. Pierre n'est pas assuré contre le cambriolage, Vive la privatisation!), mais elle est plus rare. Pour un SN comme la réparation, elle paraît même quasiment inexistante (e.g. ?Cette entreprise est spécialisée dans la réparation,??Sylvie donne un cours sur la réparation). Il semble donc y avoir des prédilections d'emploi pour les nominalisations de verbes sous-déterminés. Des noms comme réparation, cambriolage, privatisation, destruction, installation, fabrication, etc. s'emploient préférentiellement pour dénoter des événements — tout au moins en l'absence d'objet exprimé. On peut se demander si ces tendances d'usage sont liées à une particularité des verbes d'origine.

\subsection{Des prédilections d'usage aux nominalisations partielles}

Certaines nominalisations de verbes sous-déterminés, contrairement aux noms qui viennent d'être examinés, ne privilégient pas l'acception occurrentielle. Tel est le cas par exemple de lecture et écriture.

L'aspect [ \pm délimité] de leurs verbes d'origine dépend effectivement de l'objet, puisqu'on dit lire (un livre / *des livres) en deux heures, écrire (une lettre / *des lettres) en vingt minutes. A l'instar de réparation, cambriolage, etc., lecture et écriture sont en principe sous-déterminés quant à leur interprétation comme occurrence ou activité. De fait, avec une structure argumentale, les deux emplois sont permis :

a. La lecture du testament aura lieu cet après-midi

b. La lecture de romans policiers compte parmi ses activités favorites

a. Pendant l'écriture de sa thèse, Sophie sortait très peu

b. L'auteur se consacre désormais à l'écriture de romans historiques

Cependant, contrairement aux SN du type la réparation, sans argument exprimé, la lecture et l'écriture n'affichent pas de prédilection pour la dénotation d'événements. L'interprétation générique d'activité est régulièrement permise :

(39) a. Sophie est une grande adepte de (la lecture / l'écriture)

b. (La lecture / l'écriture) est au cœur de l'apprentissage d'une langue étrangère

c. On encourage (la lecture / l'écriture) dès le plus jeune âge 
On peut même penser que cette interprétation domine dans l'usage du nom. L'écriture en particulier, sans expansion, désigne difficilement un événement (e.g. ?L'écriture a été reportée au mois prochain, ?Après l'écriture, Pierre a regardé un film). La prédilection d'usage est donc l'inverse de celle observée pour réparation, cambriolage, etc.

Ces différentes tendances correspondent-elles à des spécificités verbales? On remarque que les noms qui favorisent l'interprétation générique sont issus de verbes qui s'emploient régulièrement sans objet, avec valeur d'activité. Ainsi est-il plus facile de dire (40a) que (40b) :
a. Pierre est en train de (lire / écrire)
b. ?Pierre est en train de (réparer / cambrioler)

L'interprétation du SN (la lecture/ l'écriture) sans exp. comme activité est conforme à l'emploi intransitif du verbe correspondant, dont il est la nominalisation. Inversement, réparer et cambrioler, qui s'emploient plus difficilement intransitivement, et qui en ce sens ne favorisent pas la lecture d'activité, conduisent plutôt à des nominalisations événementielles.

Notre hypothèse est la suivante: les verbes qui ont un emploi intransitif établi encouragent la nominalisation d'activité. Corollairement, plus le verbe s'emploie difficilement intransitivement, plus sa nominalisation a tendance à dénoter une occurrence.

Cette hypothèse se confirme pour un verbe comme bricoler. Ce verbe, transitif (e.g. bricoler (une installation / un moteur / un jouet)), s'emploie aisément intransitivement, avec la lecture d'activité :

Sylvain est en train de bricoler

Et la nominalisation correspondante est un nom d'activité, semblable à ceux analysés au § 3.1. (e.g. faire du bricolage, deux heures de bricolage, Pierre adore le bricolage). L'emploi intransitif du verbe détermine donc la nature sémantique de la nominalisation.

Il y a cependant une différence notable avec les cas précédents (lecture, écriture) : la nominalisation d'occurrence est ici exclue. En effet, contrairement à lecture et écriture, bricolage n'accepte pas les compléments en de dénotant un argument interne. On peut comparer les exemples (37a), (38a) et :
a. ??Le bricolage de l'installation a eu lieu à l'aube
b. ??Le mécanicien s'est blessé pendant le bricolage du moteur
c. ??Le bricolage du jouet m'a demandé du temps

Bricolage ne peut pas être la nominalisation de l'emploi transitif de bricoler ; a fortiori, il se cantonne à l'interprétation d'activité associée à l'emploi intransitif. Il y a ici un certain décalage entre le verbe et sa nominalisation, car le nom n'est dérivé que d'une partie des acceptions du verbe. Nous parlerons en l'occurrence de nominalisation partielle.

On trouve d'autres exemples de nominalisations partielles. Tel est le cas d'admiration et d'ignorance. Admirer et ignorer ont deux acceptions, dont l'une est dynamique (43a) et l'autre statique (43b) :

a. Vincent (admire le paysage / ignore les convives)

b. Vincent (admire le courage de son frère / ignore le nom de cette plante)

Cette polysémie apparait clairement dans la compatibilité avec être en train de et la possibilité de reprise par faire : 
(44) a. Vincent est en train (d'admirer le paysage / d'ignorer les convives)

b. *Vincent est en train (d'admirer le courage de son frère / d'ignorer le nom de cette plante)

a. Vincent (admire le paysage / ignore les convives) et Alice fait pareil

b. *Vincent (admire le courage de son frère / ignore le nom de cette plante) et Alice fait pareil

Cependant, les nominalisations abstraites d'admirer et d'ignorer excluent l'acception dynamique. En effet, admiration et ignorance rejettent la structure argumentale dynamique (avec un complément d'agent introduit par par) correspondant aux emplois du type (43a) :
a. *l'admiration du paysage par Vincent
b. *l'ignorance des convives par Vincent

Ils ne peuvent pas se combiner avec un verbe support dynamique (*(procéder à / effectuer / réaliser) (une admiration / une ignorance), *faire (de l'admiration / de l'ignorance)), ni s'employer avec la locution en cours (*(l'admiration / l'ignorance) en cours, *en cours (d'admiration / d'ignorance)). En outre, admiration et ignorance n'ont pas de dénotation événementielle :

*Quelle est la date de (l'admiration / l'ignorance) ?

a. *L'admiration a eu lieu au crépuscule

b. *L'ignorance a eu lieu dans le jardin
a. *Vincent s'est extasié pendant l'admiration

b. *Vincent a lu pendant l'ignorance

Par contre, l'interprétation statique de ces deux noms est tout à fait régulière. Ignorance peut en effet s'employer dans les constructions locatives typiques des noms d'états (Cette population est plongée dans l'ignorance) et dans des génitifs de qualité (un homme d'une grande ignorance) (cf. Van de Velde 1995). De son côté, admiration est un nom de sentiment, puisqu'il peut figurer en position de $\mathrm{N}$ dans les expressions de la forme le $N$ de $x$ pour $y, X$ (a / éprouve / ressent) du $N$ pour $y$ et $Y$ suscite le $N$ (de $x$ ) (cf. Flaux \& Van de Velde 2000) :
a. l'admiration de Vincent pour son frère
b. Vincent (a / éprouve / ressent) de l'admiration pour son frère
c. Son frère suscite l'admiration

Admiration et ignorance sont donc des nominalisations partielles d'admirer et ignorer, correspondant uniquement à leur acception statique. Ils n'héritent pas de la polysémie de leurs verbes d'origine.

\section{Conclusion}

L'aspect des nominalisations verbales est souvent calqué sur celui des verbes correspondants. Les traits aspectuels $[ \pm$ dynamique], $[ \pm$ délimité], $[ \pm$ duratif] se transmettent régulièrement aux noms déverbaux abstraits. Du verbe au nom, l'héritage aspectuel est non seulement permis, mais également répandu. Par conséquent, les catégories aspectuelles distinguées dans le domaine verbal s'appliquent aussi dans le domaine nominal.

Le calquage aspectuel du nom sur le verbe n'est cependant pas toujours avéré. Nous avons constaté l'existence de décalages de différents types. Pour certains noms, comme discussion, manifestation et promenade, le trait [- délimité] de la base n'est pas respecté : au verbe d'activité correspond une nominalisation occurrentielle, susceptible de dénoter des événements. Pour d'autres noms, comme bricolage, admiration et ignorance, la nominalisation est partielle, au sens où elle ne reprend pas tout 
l'éventail aspectuel du verbe. L'infidélité aspectuelle consiste alors, non pas en un simple changement de spécification d'un trait donné, mais en un défaut de sens. La polysémie du verbe de base n'est pas nécessairement transmise au nom abstrait correspondant.

Les décalages aspectuels constatés peuvent conduire à préciser certaines notions couramment employées dans le domaine verbal. En particulier, la distinction entre noms d'événements issus de prédicats d'accomplissement et noms d'événements dérivés de verbes d'activité pose la question de savoir comment, dans le domaine nominal, s'applique le critère de la télicité. Un nom dont on pourrait dire qu'il est télique doit-il impliquer la visée d'un terme final, marquant la réalisation du procès décrit? Ou le simple bornage de l'action, fût-elle homogène, suffit-il ? Comme nous l'avons vu, il est nécessaire de distinguer, pour les noms tout au moins, entre les événements bornés mais non culminants et les événements qui impliquent un climax déterminant la fin de l'action.

L'étude des nominalisations peut également révéler certaines particularités sémantiques des bases verbales. Nous avons suggéré ailleurs que les verbes d'activité qui conduisent à des nominalisations événementielles se distinguent des autres verbes d'activité par leur tendance à décrire des actions isolées (cf. Haas \& Huyghe à paraître). De même, la mise en évidence de prédilections d'emploi différentes pour des noms comme réparation et lecture peut étayer l'hypothèse selon laquelle réparer et lire, dont l'aspect dépend apparemment de l'objet, connaissent des différences aspectuelles profondes. On peut penser en effet, au vu des propriétés distinctives de réparation et de lecture, que réparer, contrairement à lire, décrit un procès qui intrinsèquement contient un objectif de réalisation. La nominalisation révèlerait ainsi une orientation sémantique profonde du verbe, au-delà de son apparente sous-détermination aspectuelle orientation d'accomplissement pour réparer et d'activité pour lire.

D'autres types de glissements aspectuels restent à étudier. Comment par exemple un verbe comme imaginer, qui est dynamique et qui décrit sans difficulté des situations particulières, conduit-il à imagination, qui ne dénote ni des événements ( ${ }^{*}$ ' 'imagination a eu lieu hier) ni une activité à proprement parler (?deux heures d'imagination, ??faire de l'imagination), mais plutôt une faculté de l'esprit (e.g. Cet enfant a beaucoup d'imagination)? Comment énerver, qui a une double lecture agentive et non agentive (Pierrot essaie d'énerver Marion vs Cette situation m'énerve), conduit-il à énervement, qui n'est en aucun cas un nom d'action (*l'énervement de Marion par Pierrot)? L'analyse de ces différents glissements sémantiques devrait nous permettre d'affiner une grille de classification qui, à ce stade, reste lacunaire.

\section{Références}

Alexiadou, A. (2001). Functional Structure in Nominals : Nominalization and Ergativity. Amsterdam / Philadelphia : John Benjamins.

Anscombre J.-C. (2005). Les deux périphrases nominales un $N$ en train / un $N$ en cours : essai de caractérisation sémantique. In Bat-Zeev Shyldkrot, H. \& Le Querler, N. (éds). Les périphrases verbales, Amsterdam: John Benjamins, 103-117.

Anscombre, J.-C. (2007). Les indicateurs aspectuels de déroulement processif : en cours de, en passe de, en train de, en voie de. Cahiers de Lexicologie, 90, 41-74.

Bach, E. (1986). The Algebra of Events. Linguistics and Philosophy, 9, 5-16.

Berthonneau, A.-M. (1989). Composantes linguistiques de la référence temporelle. Les compléments de temps, du lexique à l'énoncé. Thèse d'état. Université de Paris VII.

Carlson, G. (1980). Reference to Kinds in English. New York / London : Garland.

Comrie, B. (1976). Aspect, an Introduction to the Study of Verbal Aspect and Related Problems. Cambridge: Cambridge University Press.

Dowty, D. (1979). Word Meaning and Montague Grammar. Dordrecht : Reidel Publishing Company. 
Flaux, N. \& Van de Velde, D. (2000). Les noms en français : esquisse de classement. Paris : Ophrys.

Ghiglione, R. et al. (1990). Prédications d'état, de déclaration et d'action: essai de classification en vue d'une application en analyse de contenu. Langages, 100, 81-100.

Giry-Schneider, J. (1978). Les nominalisations en français : l'opérateur "faire" dans le lexique, Genève : Droz.

Grimshaw, J. (1990). Argument Structure. Cambridge, MA : The MIT Press.

Gross, G. (1996). Prédicats nominaux et compatibilité aspectuelle. Langages, 121, 54-72.

Haas, P. \& Huyghe, R. (à paraître). Les propriétés aspectuelles des noms d'activités.

Heyd, S. \& Knittel, M.L. (2006). Quelques remarques à propos des noms d'activités. Rencontres linguistiques du Grand Est, 13-14 juin 2006.

Huyghe, R. (2006). Les noms génériques d'espace en français. Thèse de doctorat, Université de Lille 3.

Huyghe, R. \& Marin, R. (2007). L’héritage aspectuel des noms déverbaux en français et en espagnol. Faits de Langues, 30, 265-274.

Jacquey, E. (2006). Cas de «polysémie logique»: modélisation de noms d'action en français ambigus entre processus et artefact. TAL 47(1), 137-166.

Langacker, R.W. (1991). Noms et verbes. Communication, 53, 103-153.

Mourelatos, A. (1978). Events, Processes and States. Linguistics and Philosophy, 2, 415-434.

Nicolas, D. (2002). La distinction entre noms massifs et noms comptables. Louvain / Paris : Editions Peeters.

Osswald, R. (2005). On Result Nominalization in German. Proceedings of Sinn und Bedeutung, 9, 256-270.

Pelletier, F.J. (1979). Non-singular reference: some preliminaries. In Pelletier, F. J. (éd.), Mass Terms : Some Philosophical Problems. Dordrecht : Reidel Publishing Company, 1-14.

Quine, W. O. (1960) [1977]. Le mot et la chose. Paris : Flammarion.

Rothstein, S. (2004). Structuring Events. A Study in the Semantics of Lexical Aspect. Oxford : Blackwell Publishing.

Smith, C. (1991). The parameter of Aspect. Dordrecht : Kluwer.

Smith, C. (1999). Activities : states or events ? Linguistics and Philosophy, 22, 479-508.

Van de Velde, D. (1995). Le spectre nominal : des noms de matières aux noms d'abstractions. Louvain / Paris : Editions Peeters.

Van de Velde, D. (1997). Un dispositif linguistique propre à faire entrer certaines activités dans des taxinomies : Faire $+d u+$ Nom d'activité. Revue de Linguistique Romane, 243-244, 369-395.

Vendler, Z. (1967). Linguistics in Philosophy. Ithaca N.Y. : Cornell University Press.

Verkuyl, H. (1971). On the Compositional Nature of the Aspects. PhD, Universite d'Amsterdam.

Verkuyl, H. (1989). Aspectual classes and aspectual composition . Linguistics and Philosophy, 12, 39-94.

\footnotetext{
${ }^{1}$ Cf. Osswald 2005, Jacquey 2006.

${ }^{2}$ La réciproque n'est pas établie. On distingue en effet entre états permanents, ou plus exactement atemporels, i.e. qui ne s'inscrivent pas dans le temps, et états épisodiques, qui peuvent être bornés dans le temps (cf. Carlson 1980, Van de Velde 1995). Le test suggéré ici est pertinent pour les premiers, mais il n'est peut-être pas probant pour les seconds. En d'autres termes, il n'est pas certain que tout nom qui valide une des trois conditions sous (1) soit dynamique. Nous reviendrons sur ce point dans nos prochains travaux, et notamment sur la question de savoir si les états épisodiques intègrent une composante aspectuelle dynamique.
} 
ISBN 978-2-7598-0358-3, Paris, 2008, Institut de Linguistique Française

Sémantique

DOI $10.1051 / \mathrm{cmlf08} 108$

\footnotetext{
${ }^{3}$ Lorsque l'objet est indéfini singulier, la lecture non générique non spécifique est possible (e.g. Pierre a cambriolé un (certain) appartement vs Quand on cambriole un appartement, cela dure en moyenne huit minutes). L'action n'en est pas moins intrinsèquement délimitée. Le même phénomène s'observe pour la nominalisation (Pierre a organisé le cambriolage d'un (certain) appartement vs Le cambriolage d'un appartement dure en moyenne huit minutes). Dans tous les cas, le SN décrit un procès délimité, que l'objet soit ou non spécifique ; il peut donc dénoter un événement, fût-il virtuel (Le cambriolage d'un appartement a eu lieu hier soir dans le voisinage vs Quand a lieu le cambriolage d'un appartement, les voisins entendent rarement quelque chose). En dépit de l'apparente généricité du SN lorsque l'argument est non spécifique, il n'y a rien de comparable ici aux cas où l'objet est indéfini pluriel, et où l'action est intrinsèquement non délimitée (e.g. *Le cambriolage d'appartements dure en moyenne huit minutes, *Quand a lieu le cambriolage d'appartements, les voisins entendent rarement quelque chose).
} 